\title{
Detection and measurement of the Dzyaloshinskii-Moriya interaction in double quantum dot systems
}

\author{
Sucismita Chutia, Mark Friesen, and Robert Joynt \\ Department of Physics, University of Wisconsin-Madison, Madison, WI 53706-1390
}

(Dated: 04/28/2006; Received textdate; Revised textdate; Accepted textdate;

Published textdate)

\begin{abstract}
Spins in quantum dots can act as the qubit for quantum computation. In this context we point out that spins on neighboring dots will experience an anisotropic form of the exchange coupling, called the Dzyaloshinskii-Moriya (DM) interaction, which mixes the spin singlet and triplet states. This will have an important effect on both qubit interactions and spin-dependent tunneling. We show that the interaction depends strongly on the direction of the external field, which gives an unambiguous signature of this effect. We further propose a new experiment using coupled quantum dots to detect and characterize the DM interaction.
\end{abstract}

PACS numbers: 73.21.La, 75.30.Et, 73.61.Ga, 03.67.Lx

Keywords: exchange interaction, quantum computing 
Solid state devices show great promise for scalable quantum information processing. Several well known proposals for quantum computing have been presented, including semiconducting quantum dots $\frac{1}{2}$ and superconducting Josephson junctions. ${ }^{2}$ Quantum dots currently enable the confinement and control of electrons on the scale of tens of nanometers, even down to the limit of one electron. ${ }^{3.4}$ Detection techniques allow the measurement of a single electron spin..$^{-}$

The solid state matrix provides both opportunities and challenges for quantum control and decoherence, due to the complex environment of the qubits. In this paper, we focus on a prominent issue for many solid state qubit implementations: the spin orbit interaction, which couples spin and charge fluctuations. As typical for qubit interactions, the spin orbit coupling can be both useful.6.7 and detrimental.8.9 Here, we consider how the spin orbit coupling affects the time evolution of two-qubit interactions in spin-based quantum dot qubits. We find that failure to account for spin orbit coupling can lead to serious control errors in the quantum computation.

The main interaction between spin qubits is the exchange coupling, which can be controlled with electronic gates, by raising or lowering the electrostatic tunnel barrier between neighboring quantum $\operatorname{dots}^{1}$ or by varying the relative depth of the wells constituting the double dot. ${ }^{10}$ Ignoring the crystal matrix, the exchange coupling would be of the Heisenberg type, with global $S U(2)$ spin symmetry: $H_{\text {Heis }}=J \mathbf{S}_{1} \cdot \mathbf{S}_{2}$. Here, $\mathbf{S}_{i}$ are spin operators and $J$ is the tunable exchange coupling constant. The presence of spin-orbit interaction introduces anisotropy into the exchange coupling, with an antisymmetric component known as the Dzyaloshinskii-Moriya (DM) interaction. ${ }^{11.12}$ Kavokin has shown that the DM exchange term also occurs in quantum dots, as a consequence of tunneling coupling. 13 Devitt et al. have proposed methods to determine its magnitude. ${ }^{14}$ Several other authors have also studied the importance of the DM interaction for quantum dot quantum computing. ${ }^{6,15,16,17,18}$

For a single pair of dots, we can write the DM interaction as $H_{\mathrm{DM}}=\alpha J \hat{\mathbf{r}} \cdot\left(\mathbf{S}_{1} \times \mathbf{S}_{2}\right)$, where $\hat{\mathbf{r}}$ is the unit vector joining the two spins. Thus, the presence of a DM interaction reduces the spin symmetry to a global $U(1)$ cylindrical symmetry, where only rotations about $\hat{\mathbf{r}}$ remain as symmetry operations. Kavokin has considered the magnitude of the coefficient $\alpha$, computing the exchange integral for the two electrons taking into account the admixture of spin projections caused by the spin-orbit interaction. 13 This calculation applies to the case when the energy levels of the individual dots are approximately equal $\left(\left|\epsilon_{1}-\epsilon_{2}\right| \lesssim J\right)$ 
and the Heitler-London method is valid. Here, we consider only this particular case. In GaAs, the predominant spin-orbit coupling is of the Dresselhaus type. For quantum dots in a $100 \AA$ GaAs quantum well, Kavokin finds $\alpha \approx 0.1$, not a particularly small value. In such a case, we expect the DM contribution to the exchange coupling will be readily apparent. For silicon dots, the Dresselhaus interaction is not present, and the predominant spin-orbit coupling arises from the Rashba interaction.

Petta et al. have recently performed a set of experiments with coupled spins in a double quantum dot system in GaAs that demonstrate control of the exchange coupling. 10 In these experiments, a qubit was defined by $|S\rangle$ and $\left|T_{0}\right\rangle$ : the singlet, and one component of the triplet states of the two-spin system respectively. Neglecting additional couplings, we would expect the Heisenberg term term to split $|S\rangle$ and $\left|T_{0}\right\rangle$, thus enabling exchange-based qubit rotations. 10 However, inhomogeneous nuclear fields and the DM interaction also mix in $\left|T_{+}\right\rangle$ and $\left|T_{-}\right\rangle$, the two other components of the triplet. The resulting loss of wavefunction probability from the qubit subspace constitutes leakage and it can be interpreted as dephasing or decoherence. However, the dynamics are actually coherent. It may therefore be possible to utilize the DM dynamics in a beneficial way, ${ }^{6.19}$ or to undo them using time-symmetric pulse shapes ${ }^{15,16,17}$ or spin echo techniques. Here, we investigate in detail the DM dynamics of a double quantum dot system, specifically considering the experiments of Petta et al. We explore how the DM interaction modifies the usual interpretation of such experiments, and we propose further experiments to detect the presence of the DM interaction and to measure its magnitude.

The Hamiltonian for our double-dot system is

$$
H=J \mathbf{S}_{1} \cdot \mathbf{S}_{2}+\alpha J \hat{\mathbf{r}} \cdot\left(\mathbf{S}_{1} \times \mathbf{S}_{2}\right)-g^{*} \mu_{B}\left(\mathbf{B}_{1} \cdot \mathbf{S}_{1}+\mathbf{B}_{2} \cdot \mathbf{S}_{2}\right)
$$

where $\mathbf{B}_{1}=\mathbf{B}_{\text {ext }}+\mathbf{B}_{n, 1}$ and $\mathbf{B}_{2}=\mathbf{B}_{\text {ext }}+\mathbf{B}_{n, 2}$. Here, $\mathbf{B}_{n, i}$ is the semiclassical field that is used to approximate the effective nuclear field for coupling of the electron spin to local nuclei in dot $i, \frac{20}{,}$ given by

$$
\mathbf{B}_{n, i}=\frac{A v_{o}}{-g^{*} \mu_{B}} \sum_{k}\left|\psi_{0}^{i}\left(\mathbf{r}_{k}\right)\right|^{2} \mathbf{I}^{k}
$$

where $\mathbf{I}^{k}$ is the nuclear spin operator for a nucleus of total spin $I$ at the lattice site $k, v_{0}$ is the volume of a unit cell containing one nuclear spin, $A$ is the hyperfine coupling strength and $\psi_{0}^{i}\left(\mathbf{r}_{k}\right)$ is the single particle envelope function for the orbital state $i$ evaluated at site $k$. 
We assume that $\mathbf{B}_{n, i}$ has a Gaussian distribution with mean zero, and a typical variance of $\sigma=2.3 \mathrm{mT}$. In the calculations reported below, our results are averaged over the distribution

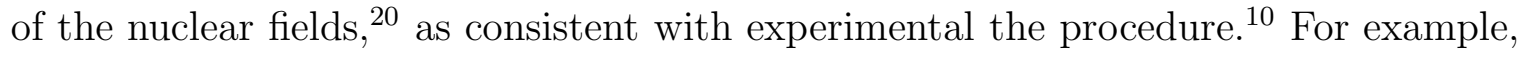

$$
\begin{array}{r}
\bar{F}=\left(2 \pi \sigma^{2}\right)^{-3 / 2} \int_{0}^{2 \pi} d \phi \int_{0}^{\pi} d \theta \sin \theta \times \\
\int_{0}^{\infty} d B_{n} B_{n}^{2} e^{-B_{n}^{2} / 2 \sigma^{2}} F\left(\mathbf{B}_{n}\right) .
\end{array}
$$

High dimensional integrals are evaluated numerically using a simple Monte Carlo integration code.

In Fig. 1, we show the appropriately averaged eigenvalues of $H$, as a function of the applied field $B_{\text {ext }}$. (Note that we use $\alpha=0.5$ in this figure. This large value of $\alpha$ is chosen only for purposes of illustration. Elsewhere in the paper we use the more physical value $\alpha=0.1$.) We observe mixing of the unperturbed singlet and triplet states at special fields. Near $B_{\text {ext }}=0$, there is mixing of the triplet states, primarily due to inhomogeneous nuclear fields $\underline{\underline{10}}$. At nonzero fields, there is an additional mixing of the singlet and triplet states, which arises from both inhomogeneous nuclear fields and the DM interaction. The mixing occurs near the resonance condition $g^{*} \mu_{B} B_{\text {ext }} \approx \pm J$, corresponding to $B_{\text {ext }} \approx \pm 0.04 \mathrm{~T}$ in the figure. From the point of view of experimental detection, a crucial point is that the mixing effect is anisotropic. This is seen clearly in Fig. 1(b) where we plot the overlaps between the eigenstates of Eq. (11) and the pure spin singlet.

We now compute the time evolution of the two coupled spins for several experimental situations of interest. The Hamiltonian in Eq. (11) has four eigenstates $\left|\psi_{1 . .4}\right\rangle$, with the corresponding eigenvalues $E_{1.4}$. For an arbitrary initial state given by $|\Psi(0)\rangle=\sum a_{i}\left|\psi_{i}\right\rangle$, we can compute the probability $P_{s}(t)=|\langle S \mid \Psi(t)\rangle|^{2}$ that this state will evolve to a spin singlet after time $t$. We consider the initial state $|\Psi(0)\rangle=|S\rangle$. The probability that the spin system will remain in its singlet state is then given by

$$
P_{s}(t)=\sum_{i=1}^{4}\left|a_{i}\right|^{4}+2 \sum_{i<j}\left|a_{i}\right|^{2}\left|a_{j}\right|^{2} \cos \left[\left(E_{i}-E_{j}\right) t / \hbar\right] .
$$

Leakage can occur due to both the DM interaction and the inhomogeneous nuclear fields. When $J$ is exponentially suppressed, leakage is due entirely to the nuclear fields. For nonvanishing $J$, the initial singlet state would remain stationary if not for the nuclear and DM mechanisms. Both mechanisms then play a role in leakage. In Fig. 2, we plot $P_{s}(t)$ 


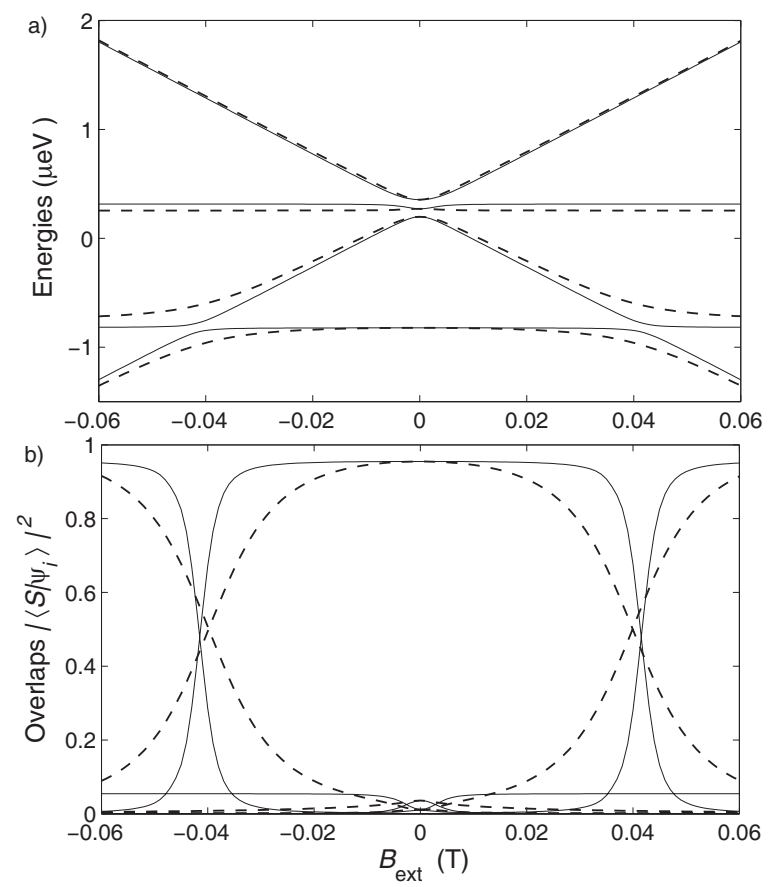

FIG. 1: (a) Energy eigenvalues of the spin Hamiltonian, Eq. (11), as a function of $B_{\text {ext }}$, for the parameters $J=1 \mu \mathrm{eV}, \alpha=0.5$ and $\sigma=2.3 \mathrm{mT}$. Here the solid line corresponds to $\mathbf{B}_{\text {ext }} \| \hat{\mathbf{r}}$ and the dashed line corresponds to $\mathbf{B}_{\text {ext }} \perp \hat{\mathbf{r}}$. (b) The overlap $\left|\left\langle S \mid \psi_{i}\right\rangle\right|^{2}$ of the energy eigenstates with the spin singlet as a function of field. Solid and dashed lines have the same meaning as in (a).

obtained after allowing the system to evolve over a "waiting time" $t=\tau_{s}$. At the resonance condition $J=g^{*} \mu_{B} B_{\text {ext }}, P_{s}$ is strongly suppressed compared to smaller and larger fields. A similar suppression of $P_{s}$ is expected in the absence of spin-orbit coupling. However, the DM relaxation mechanism exhibits a strong dependence on the orientation of $\mathbf{B}_{\text {ext }}$ with respect to $\hat{\mathbf{r}}$, which cannot be explained by nuclear fields. This dependence on field orientation provides an important signature of the DM interaction.

In the experiments of Petta et al., an initial singlet state is prepared with both electrons in a single quantum dot. The electrons are subsequently separated into two dots while retaining their singlet correlations. A waiting time ensues, consistent with the analysis presented above, after which the singlet probability $P_{s}$ is measured. The data can be fit using a semiclassical model, ${ }^{21}$ obtaining a dephasing time of about $T_{2}^{*} \approx 10 \mathrm{~ns}$ and effective static nuclear field $B_{\text {nuc }}=2.3 \mathrm{mT}$. This $T_{2}^{*}$ is an ensemble averaged time for relaxation to the asymptotic value. More relevant for quantum information is the short-time behavior, 

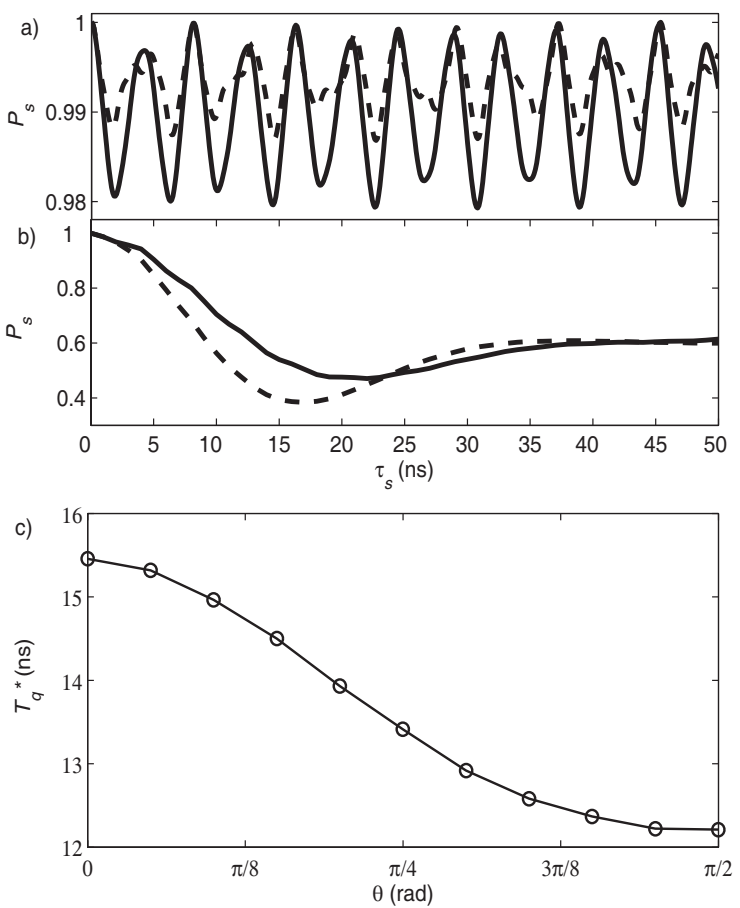

FIG. 2: (a,b) The probability $P_{s}$ of an initial spin singlet to remain in the singlet state as a function of the "waiting time" $\tau_{s}$ for $J=1 \mu \mathrm{eV}, \alpha=0.1$ and $\sigma=2.3 \mathrm{mT}$. Solid lines correspond to the field orientation $\mathbf{B}_{\text {ext }} \| \hat{\mathbf{r}}$ and dashed lines correspond to $\mathbf{B}_{\text {ext }} \perp \hat{\mathbf{r}}$. (a) $B_{\text {ext }}=100 \mathrm{mT}$, (b) $B_{\text {ext }}=40 \mathrm{mT}$ (resonance condition), (c) Dephasing time $T_{q}^{*}$, obtained from a fit to a parabolic equation as a function of the orientation angle $\theta$ between $\mathbf{B}_{\text {ext }}$ and $\hat{\mathbf{r}}$. The fitting data correspond to the curves $B_{\text {ext }}=40 \mathrm{mT}$ in (b).

characterized by the quantity $T_{q}^{*}$, defined by $P_{s}(\tau) \cong 1-\left(\tau / T_{q}^{*}\right)^{2}$. In Fig. $2(\mathrm{c}), T_{q}^{*}$ is plotted as a function of $\theta$, the angle between $\mathbf{B}_{\text {ext }}$ and $\hat{\mathbf{r}}$. For $B_{\text {ext }}=40 \mathrm{mT}$, the results show a significant dependence on $\theta$. Note that the $t^{2}$ dependence of $P_{s}$ (as opposed to an exponential decay) is due to an absence of dissipation in our model.

The significance of the DM interaction becomes most apparent during exchange gate operations, when the Heisenberg and DM couplings, $J$ and $\alpha J$ respectively, are non-vanishing. We consider the "Rabi oscillation" experiment of Petta et al., in which the spins are initially prepared in the state $|n\rangle$, corresponding to the ground state determined by the nuclear fields when $J=0$. The initial state is not an eigenstate of $H_{\text {Heis }}$, so when the Heisenberg interaction is initiated, coherent oscillations will occur between the singlet and triplet manifolds. 
Thus, after an exchange period of $\tau_{E}=2 \pi \hbar / J$, the spins will return to their initial state. Both inhomogeneous nuclear fields and the DM interaction affect this picture by mixing in the different triplet states inhomogeneously, causing $P_{n}(t)=|\langle n|U(t)| n\rangle|^{2}$ to decay. Here, $P_{n}$ is the probability to return to the initial state $|n\rangle, 22$ and $U(t)$ is the unitary evolution operator for the spin Hamiltonian. If we define the $a_{i}$ coefficients of the initial state as $|n\rangle=\sum a_{i}\left|\psi_{i}\right\rangle$, then $P_{n}(t)$ is given by Eq. (4).

We have computed $P_{n}(t)$ for experimental parameters consistent with Ref. [10]. The results are shown in Fig. 3. Here, the external field is much larger than the nuclear field, so the initial state of the evolution is nearly spin polarized. The exchange coupling is then switched suddenly to a value slightly off from the resonant condition $J=g^{*} \mu_{B} B_{\text {ext }}$ for a period $\tau_{E}$. We plot two cases: with and without the DM interaction $(\alpha=0.1,0$, respectively). In both cases, the initial state $|\Psi(0)\rangle \approx\left|T_{+}\right\rangle$is very similar to the ground eigenstate. (Recall that the nuclear fields and the DM interactions cause a hybridization of the $|S\rangle$ and $\left|T_{+}\right\rangle$states near their level crossing. But away from the crossing, the eigenstates retain their $|S\rangle$ and $\left|T_{+}\right\rangle$character.) Therefore, in the long-time limit $\tau_{E} \gg \tau_{\text {nuc }}, P_{n}$ does not deviate greatly from 1 . Here, $\tau_{\text {nuc }} \approx \hbar / g^{*} \mu_{B} B_{\text {nuc }}$ is the nuclear mixing time. We note that the solution including the DM interaction is clearly distinguishable from the $\alpha=0$ case. This is because the DM coupling enhances the hybridization of $|S\rangle$ and $\left|T_{+}\right\rangle$, and thus the difference between the initial and final states.

Another obvious feature in Fig. 3 is the initial rapid oscillations of $P_{n}$. Since the initial spin state is not an eigenstate of the exchange Hamiltonian, it can undergo coherent oscillations prior to nuclear mixing. In the figure, the predominant oscillations occur between the $S$-like and $T_{+}$-like states, with an approximate energy splitting of $g^{*} \mu_{B} B_{\text {ext }}-J$ and a corresponding oscillation period of $2 \pi \hbar /\left(g^{*} \mu_{B} B_{\text {ext }}-J\right)$. Note that without any true damping mechanisms the curves are subject to Poincaré recurrence and will return to 1.

We now propose an experiment to unambiguously detect the presence of the DM interaction. In Fig. 3, the suppression of $P_{n}$ was strongly enhanced by DM interactions near resonance $(2.5 \mu \mathrm{eV})$. So we perform the previous experiment in a large external field where we can tune the exchange coupling to its resonant condition $J=g^{*} \mu_{B} B_{\text {ext }}$ during the exchange evolution. Under these conditions, the hybridization of $|S\rangle$ and $\left|T_{+}\right\rangle$is maximized, so that the initial and final states will be quite different. Consequently, after nuclear mixing, $P_{n}$ approaches 0.5 . The reason for choosing $B_{\text {ext }}$ (and thus $J$ ) to be large is that this allows 


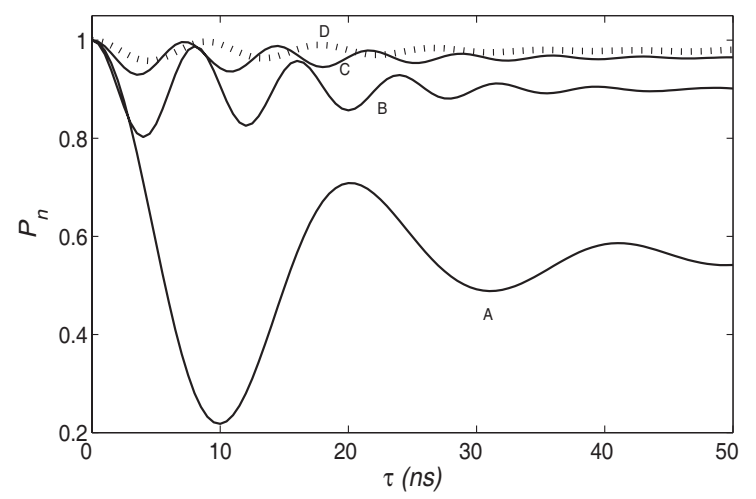

FIG. 3: Coherent oscillations of $P_{n}$, corresponding to the "Rabi oscillations" of Ref. [10] at $B=100 \mathrm{mT}$, and $\sigma=2.3 \mathrm{mT}$. Solid curves include the effects of the DM interaction. The dotted curve corresponds to DM interactions turned off ( $\alpha=0$ ). (A) $J=2.5 \mu \mathrm{eV}, \alpha=0.1$, (B) $J=3 \mu \mathrm{eV}$, $\alpha=0.1,(\mathrm{C}) J=2 \mu \mathrm{eV}, \alpha=0.1$, (D) $J=3 \mu \mathrm{eV}, \alpha=0$.

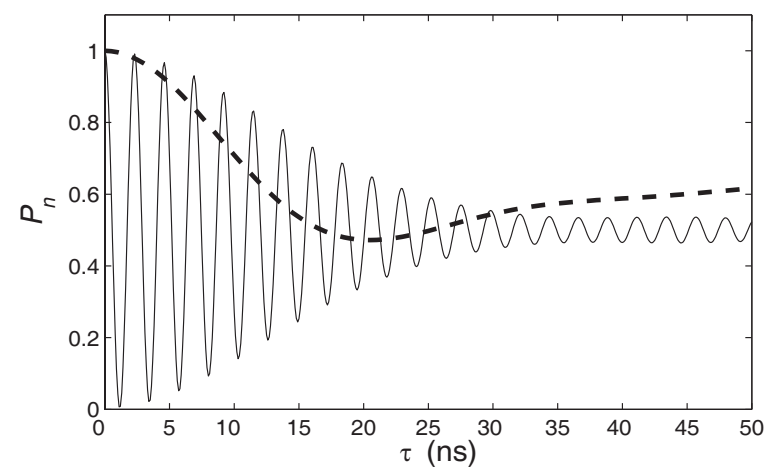

FIG. 4: Proposed experiment to observe DM interactions. The system is prepared in the initial state $\left|T^{+}\right\rangle$in a large field, $B_{\text {ext }}=1 \mathrm{~T}$. A strong exchange coupling $(J=25.5 \mu \mathrm{eV})$ is initiated at the resonance condition $J=g^{*} \mu_{B} B_{\text {ext }}$ with $\sigma=2.3 \mathrm{mT}$, producing fast oscillations dampened by nuclear mixing (solid curve). In the absence of the DM interaction, no fast oscillations are observed (dashed curve).

many coherent oscillations to occur before nuclear mixing.

Some typical results are shown in Fig. 4, with and without the DM interaction. Because a large value of $J$ has been used, the hybridization of $|S\rangle$ and $\left|T_{+}\right\rangle$is completely dominated by the DM interaction for the case $\alpha=0.1$. Rapid oscillations occur between these two 
states, with an energy splitting given by $2\left|\left\langle T_{+}\left|H_{\mathrm{DM}}\right| S\right\rangle\right|=\alpha J / \sqrt{2}$, and an oscillation period of $\pi \sqrt{8} \hbar / \alpha J$. To see the fast oscillations, the exchange coupling should be turned on quickly compared to the oscillation period, so that the initial state cannot evolve adiabatically to the ground state. Similar to Fig. 3, the oscillation envelope is eventually suppressed by nuclear mixing. However in the large $B_{\text {ext }}$ limit, the fast oscillation period is determined only by DM interactions, not hyperfine effects. This can be confirmed by plotting $\left[(\right.$ fast oscillation period $\left.) \times B_{\text {ext }}\right]$ vs. $B_{\text {ext }}$, which should remain a constant. The hyperfine effects can also be eliminated by polarizing the nuclear spins or by employing a standard Hahn spin echo sequence.

Two-qubit operations require a very accurate knowledge of the spin-spin interaction, and the DM interaction is expected to be about a $10 \%$ effect in GaAs. It is therefore very important to develop methods to measure it in double quantum dot systems. Because the interaction breaks spin rotation invariance it can be detected: its effects depend strongly on the direction of the applied field in ways that we have described. By carefully choosing external parameters, it is also possible to determine the magnitude of the DM coupling by measuring the oscillation period for evolution between the singlet and triplet states.

We gratefully acknowledge conversations with C. M. Marcus and W. O. Puttika. This work was supported by NSA and ARDA under ARO contract number W911NF-04-1-0389 and by the National Science Foundation through the ITR (DMR-0325634) and EMT (CCF0523675) programs.

1 D. Loss and D. P. DiVincenzo, Phys. Rev. A 57, 120 (1998).

2 Y. Makhlin, G. Schon, and A. Shnirman, Rev. Mod. Phys. 73, 357 (2001).

3 M. Ciorga, A. S. Sachrajda, P. Hawrylak, C. Gould, P. Zawadzki, S. Jullian, Y. Feng, and Z. Wasilewski , Phys. Rev. B 61, R16315 (2000).

4 J. M. Elzerman, R. Hanson, J. S. Greidanus, L. H. Willems van Beveren, S. De Franceschi, L. M. K. Vandersypen, S. Tarucha, and L. P. Kouwenhoven, Phys. Rev. B 67, 161308(R) (2003).

5 R. Hanson, L. H. Willems van Beveren, I. T. Vink, J. M. Elzerman, W. J. M. Naber, F. H. L. Koppens, L. P. Kouwenhoven, and L. M. K. Vandersypen, Phys. Rev. Lett. 94, 196802 (2005).

6 L.A. Wu and D. A. Lidar, Phys. Rev. A 66, 062314 (2002); 
7 M. Friesen, C. Tahan, R. Joynt, and M. A. Eriksson, Phys. Rev. Lett. 92, 037901 (2004).

8 D. Mozyrsky, S. Kogan, V. N. Gorshkov, G. P. Berman, Phys. Rev. B 65, 245213 (2002)

9 C. Tahan, M. Friesen, and R. Joynt, Phys. Rev. B 66, 035314 (2002).

10 J. R. Petta, A. C. Johnson, J. M. Taylor, E. A. Laird, A. Yacoby, M. D. Lukin, C. M. Marcus, M. P. Hanson, and A. C. Gossard, Science 309, 2180 (2005).

11 I Dzyaloshinskii, J. Phys. Chem. Solids 4, 241 (1958).

12 T. Moriya, Phys. Rev. 120, 91 (1960).

13 K. V. Kavokin, Phys. Rev. B 64, 075305 (2001).

14 S.J.Devitt, J.H. Cole, and L.C.L. Hollenberg, quant-ph/0511168 v2, (2005)

15 N. E. Bonesteel, D. Stepanenko, and D. P. DiVincenzo, Phys. Rev. Lett. 87, 207901 (2001).

16 G. Burkard and D. Loss, Phys. Rev. Lett. 88, 047903 (2002).

17 D. Stepanenko, N. E. Bonesteel, D. P. DiVincenzo, G. Burkard, and D. Loss, Phys. Rev. B 68, $115306(2003)$.

18 K. V. Kavokin, Phys. Rev. B 69, 075302 (2004).

19 D. Stepanenko and N. E. Bonesteel, Phys. Rev. Lett. 93, 140501 (2004).

20 W.A. Coish and D. Loss, PRB 72, 125337 (2005).

21 K. Schulten and P. G. Wolynes, J. Chem. Phys. 68, 3292 (1978).

22 Our $P_{n}$ corresponds to the $P_{\uparrow \downarrow}$ of Petta et al. 